\title{
A CHECKLIST OF CRUSTACEA OF ST. CROIX, VIRGIN ISLANDS
}

The present checklist on the crustacea of St. Croix is one of a series intended to cover comprehensively the natural history of this Island. It is based on extensive fieldwork with the Virgin Islands Department of Health by the writer from 1935 to 1944 . The material is deposited in the U.S. National Museum. (Specimens were preserved in a 60-percent alcohol solution.) A total of 90 genera, 132 species is recorded.

In its scope the present list includes many crustaceans not recorded in the writer's previous paper "The Brachyuran Crabs of St. Croix" which, through force of circumstances, was prepared as a separate.

Crustaceans are found in a remarkably wide variety of situations. Accordingly, collecting techniques varied and were often novel. A variety of nets and fishtraps was employed. Much of the marine material could not have been taken without the cooperation of individual deep-sea fishermen. Whenever the fishtraps were set deeper than 4 fathoms it was necessary to haul them up speedily and dump the contents into the boat to intercept the more active crabs and shrimps. Another method used employed crushed bait, usually a large crab, to attract crabs into the shallows.

Minute crustaceans that remained concealed in clusters of seaweed and blocks of dead coral were flushed out by placing the material in basins of fresh water. Insect nets of coarse meshbags were most useful for collecting in streams. By pushing the net under the submerged weeds overhanging the banks the shrimps are forced into the trap.

The Island of St. Croix lies between the 17 th and 19th parallels north latitude, and $64^{\circ} 10^{\prime}$ and $65^{\circ} 30^{\prime}$ west longitude. The Island is 21 miles in length and 5 miles in greatest width on the western portion, tapering wedgelike to a point on the eastern end. The mountain system extending the length of the Island runs parallel to the North Coast and fans out on the South side in an extensive plain. Radiating spurs form deep valleys and steep ridges, the highest elevation is Mount Eagle which rises to 1,165 feet.

Five depressions along the coasts, formerly fresh to brackish water-holding areas and the habitat of a rich variety of animal life, have been drained. Of

1 In the preparation of this paper the writer has been most generously assisted by a number of persons. Dr. Waldo I. Schmitt, Curator, Division of Marine Invertebrates, U.S. National Museum, obtained determinations of crustacea. Or. Fenner A. Chace, Jr., Senior Zoologist, Department of Invertebrate Zoology, U.S. National Museum, offered many valuable suggestions, and rendered his kind assistance in the revision of the nomenclature. Mr. J. O. Maloney determined the Tanaidacea and Isopoda, and Mr. C. R. Shoemaker the Amphipoda. Mr. T. E. Bowman checked the Maloney and Shoemaker lists. Mr. Arnold Ross, Department of Living Invertebrates, American Museum of Natural History, kindly updated the nomenclatural Order of the Isopoda and Amphipoda. 
the mangrove swamps, former prosperous wildlife sanctuaries on the Island, several have been filled in under private and Government land reclamation programs. During the past 20 years many flowing streams have dried up, and the few trickling waterways during the wet season are reduced to pools in the dry season.

Precipitation varies from 30 to 90 inches from year to year; the normal average is 46 inches annually, with irregular periods of severe drought.

\section{CHECKLIST}

Order Stomatopoda

Family Squillidae

Gonodactylus oerstedii Hansen

Pseudosquilla ciliata (Fabricius)

Order Tanaidacea

Leptochelia forresti (Stebbing)

Order Isopoda

Suborder Flabellifera

Family Cirolanidae Cirolana parva Hansen

Family Excirolanidae Excirolana mayana (Ives)

Family Excorallanidae Excorallana oculata (Hansen)

Family Cymothoidae Anilocra laticauda M. Edwards

Suborder Oniscoidea

Family Tylidae

Tylos latreilli A. \& S.

Family Armadillidiidae Cubaris murina Brandt Paracerceis caudala (Say)

Order Amphipoda

Suborder Gammaridea

Family Hyalidae Allorchesles sp.

Family Ampithoidae Cymadusa filosa Savigny

Family Gammaridae Elasmopus sp. Melila palmata (Mentagu)

Melaniphargus sp.

In dead coral, Salt River reef. Under rocks in shallows, Judith Fancy Bay. In dead corals, Salt River reef.

From seaweed in shallows, Envy Bay.

In moss on dead corals, Salt River Bay.

On moss-covered submerged mangrove branches, Fairplain Lagoon.

In moss on coral reef, Salt River Bay.

Taken from gill plates of fish, red snapper.

Under moist seaweed on beach, South Gate Bay.

From a deserted bird nest, Sight.

In moss on corals, Salt River Bay.

On moss-covered submerged mangrove branches, Fairplain Lagoon

On moss-covered submerged mangrove branches, Fairplain Lagoon.

On moss in shallows, Salt River Bay.

On moss-covered submerged mangrove branches, Fairplain Lagoon.

In a brackish water well. Frederiksted. 
Family Ampithoidae

Ampithoe megaloprotopus Stebbing

Family Talitridae

Orchestia platensis (Kroyer)

Family Atylidae

Atylus minikoi (Walker)

Family Corophiidae

Grandidierella megnae (Giles)
On moss in shallows, Salt River Bay.

Under moist seaweed on beach, South Gate.

On moss-covered submerged mangrove branches, Fairplain Lagoon.

On moss-covered submerged mangrove branches, Fairplain Lagoon; on seaweed in shallows, Envy Bay.

\section{DECAPOD CRUSTACEANS COLLECTED AT ST, CROIX BY HARRY A. BEATTY}

Order Decapoda

Suborder Natantia

Section Penaeiden

Family Penaeidae

Subfamily Penaeinae

Penaeopsis (Melapenaeopsis) smilhi Schmitt

Penaeus aztecus Ives

Section Caridea

Family Atyidae

Alya innocous (Herbst)

Potimirim americana (GuérinMéneville)

Tiphocaris elongala (GuérinMéneville)

Fanily Palacmonidae

Subfanily Palaemoniae

Macrobrachium acanthurus

(Wiegmann)

Macrobrachium carcinus (Linnaeus)

Macrobrachium faustinum (De Siaussure)

Macrobrachium sp.

Palaemon (Palaeander) northropi (Rankin)

Palaemon (Palaemon) pandaliformis (Stimpson)
Krause Lagoon.

Envy Bay, Krause Lagoon, Salt River Reef.

Upper Love, Fairplain, Caledonia, Crique, La Grange, streams, Mt. Welcome Swamp.

Mt. Welcome Swamp.

Upper Love, Fairplain, Caledonia, Bethlehem, Prosperity Garden, streams; Mt. Welcome Swamp; Morning Star Swamp; Rustoptwist Pond.

Two Williams Pond; Morning Star Swamp; Mt. Welcome Swamp.

Upper Love, Caledonia, Lebanon Hill, Windsor, streams.

Upper Love, Fairplain, Caledonia, streams; Mt. Welcome Swamp; Two Williams Pond.

Mt. Welcome Swamp.

Salt River Reef, seaweed.

Mt. Welcome Swamp. 
Subfamily Pontoniinae

Periclimenes (Harpilius) ameri- Salt River Reef, seaweed. canus (Kingsley)

Family Alpheidae Alpheus bahamensis Rankin Alpheus floridanus Kingsley Alpheus formosus Gibbes Alpheus viridari (Armstrong)

Family Hippolytidae Latreutes fucorum (Fabricius) Thor floridanus Kingsley?

Suborder Reptantia

Section Macrura

Family Palinuridae Panulirus argus (Latreille)

Family Scyllaridae Scyllarides aequinoctialis (Lund)

Section Anomura

Family Porcellanidae

Petrolisthes tridenlalus Stimpson

Family Coenobitidae

Coenobila clypealus (Herbst)

Family Diogenidae

Clibanarius cubensis (De Saussure)

Clibanarius tricolor (Gibbes)

Darlanus, sp.

Pelrochirus bahamensis (Herbst)

Family Paguridae

Pagurus marshi Benedict

Family Hippidae

Hippa cubensis (De Saussure)

Section Brachyura

Family Dromiidae

Dromia erythropus (G. Edwards)

Dromidia antillensis Stimpson

Family Homolidae

Homologenus rostralus (A.

Milne-Edwards)

Family Dorippidae

Cymonomus quadratus A. Milne-

Edwards

Family Leucosiidae

Subfamily Ebaliinae

Lithadia granulosa A. Milne-

Edwards

Family Calappidae

Subfamily Calappinae

Calappa angusia A. Milne-

Edwards

Krause Lagoon, seaweed.
Salt River Reef from Corals.

Salt River Reef in Corals.

Krause Lagoon; Salt River Reef, seaweed.

Floating gulf weed.

Salt River Reef in Corals. 
Calappa ocellala Holthuis

Calappa gallus (Herbst)

Subfamily Matutinae

Osachila antillensis Rathbun

Family Majidae

Subfamily Inachinae

Anomalothir furcillatus

(Stimpson)

Euprognatha gracilipes A.

Milne-Edwards

Stenorhynchus seticornis

(Herbst)

Subfamily Ophthalmiinae

Pitho lherminieri (Schramm)

Subfamily Acanthonychinae

Acanthonyx petiverii $\mathrm{H}$. Milne-

Edwards

Epiallus biluberculalus $\mathrm{H}$.

Milne-Edwards

Menaethiops portoricensis

Rathbun

Subfamily Pisinae

Chorinus heros (Herbst)

Trachymaia cornuta A. Milne-

Edwards

Subfamily Mithracinae

Macrococloma diplacanthum

(Stimpson)

Macrocoeloma eutheca (Stimpson)

Microphrys bicornutus (Latreille)

Mithrax (Mithrax) acuticornis Stimpson

Mithrax (Mithrax) caribbaeus

lathbun

Milhrax (Mithrax) hispidus

(Herbst)

Milhrax (Mithrax) holderi

Stimpson

Mithrax (Mithrax) pilosus

liathbun

Mithrar (Milhrax) pleuracanthus Stimpson

Milhrax (Mithraculus) coryphe (Herbst)

Withrax (Mithraculus) forceps

(A. Milne-lidwards)

Milhrax (Milhraculus) sculptus

(I,amarek) 
Teleophrys ornatus Rathbun

Family Parthenopidae

Subfamily Parthenopinae

Helerocrypla granulala

(Gibbes)

Solenolambrus tenellus Stimpson

Family Portunidae

Subfamily Portuninae

Callinectes bocourti A. MilneEdwards

Callinectes exasperatus (Gerstaecker)

Callinecles marginalus (A. Milne-Edwards)

Callinectes ornatus Ordway

Callinectes sapidus acutidens Rathbun

Porlunus (Achelous) sebae (H. Milne-Edwards)

Family Pseudothelphusidae

Epilobocera sinuatifrons (A. Milne-Edwards)

Family Xanthidae

Actaea acantha (II. Milne Edwards)

Actaea rufopunctala nodosa Stimpson

Actaea setigera (II. Milne Edwards)

Carpilius corallinus (Herbst)

Chlorodiella longimanus (H.

Milne-Edwards)

Eriphia gonagra (Fabricius)

Eurypanopeus abbrevialus (Stimpsun)

Eurytium limosum (Say)

Leplodius floridanus (Gibbes)

Micropanope barbadensis (Rathbuin)

Micropanope lobifrons A. MilneEdwards

Micropanope pusilla A. MilneEdwards

Micropanope sculplipes Stimpson Micropanope urinalor (A. MilneEdwards)

Ozius reticulalus (Desbonne and Schramm)

Panopeus bermudensis Benedict and Rathbum 
Panopeus herbstii H. Milne Edwards, forma typica

Panopeus herbstii, forma crassa A. Milne-Edwards

Panopeus herbstii, forma simpsoni Rathbun

Panopeus occidentalis De Saussure

Pilumnus marshi Rathbun

Pilumnus reticulalus Stimpson, forma fragosa A. Milne-EdEdwards

Xanthodius denticulatus (White)

Family Palicidae

Palicus affinis A. Milne-Edwards and Bouvier

Palicus angustus Rathbun

Palicus depressus Rathbun

Palicus sicus (A. Milne-Edwards)

Family Grapsidae

Subfamily Grapsinae

Geograpsus lividus (H. MilneEdwards)

Goniopsis cruentala (Latreille)

Grapsus grapsus (Linnaeus)

Pachygrapsus corrugatus (Von Martens)

Pachygrapsus gracilis (De Saussure)

Pachygrapsus transversus

((iibbes)

Sulfamily Sesarminae

Aralus pisonii (H. Milne Edwards)

Cycloyrapsus integer H. Milne Edwards

Sesarma (Holometopus) americanum J)e Saussure

Sesarma (Holometopus) ricordi H. Milne-Edwards

Subfanily Plagusiinae

Perrnon gibbesi (H. Milne-Ed-

Edwards)

Plagusia depressa (Fabricius)

Family Gecarcinidae

Cardisoma guanhumi Latreille

Gecarcinus laleralis (Freminville)

Gecarinus ruricola (Linnaeus)

Family Ocypodidae

Subfamily Ocypodinae 
Ocypode quadrala (Fabricius)

Uca (Minuca) leplodaclylus

Rathbun

Uca (Minuca) burgersi Hal thuis

Uca (Minuca) rapax (Smith)

\section{Harry A. Beally}

2254 Washington A venue

New York N.Y. 10457 